\title{
Entanglement dynamics of a strongly driven trapped atom
}

\author{
Maryam Roghani, Hanspeter Helm, and Heinz-Peter Breuer \\ Physikalisches Institut, Universität Freiburg, Hermann-Herder-Strasse 3, D-79104 Freiburg, Germany
}

(Dated: November 1, 2018)

\begin{abstract}
We study the entanglement between the internal electronic and the external vibrational degrees of freedom of a trapped atom which is driven by two lasers into electromagnetically-induced transparency. It is shown that basic features of the intricate entanglement dynamics can be traced to Landau-Zener splittings (avoided crossings) in the spectrum of the atom-laser field Hamiltonian. We further construct an effective Hamiltonian that describes the behavior of entanglement under dissipation induced by spontaneous emission processes. The proposed approach is applicable to a broad range of scenarios for the control of entanglement between electronic and translational degrees of freedom of trapped atoms through suitable laser fields.
\end{abstract}

PACS numbers: 03.67.Bg, 03.65.Yz, 37.10.De

A trapped atom driven by two laser beams under conditions of electromagnetically-induced transparency (EIT) represents a realistic model for current experiments on ion or atom trapping [1, 2. Quantum correlations between the translational and the electronic degree of freedom of the trapped atom play a crucial role in the cooling dynamics of trapped atoms and ions. Indeed, such correlations are responsible for the transfer of vibrational energy of the trapped atom into excess energy of the scattered radiation field [3].

Here, we explore the dynamical behavior of entanglement [4, 5. between the external vibrational degree of freedom of the trapped atom and its internal electronic degree of freedom during the cooling process. Solving numerically the master equation [6] which describes the dynamics of the composite state $\rho(t)$ of the trapped atomlaser field system, we investigate the evolution of quantum correlations by use of the negativity [ 7 ]

$$
\mathcal{N}(t)=\mathcal{N}(\rho(t))=\frac{1}{2}\left(\left\|\rho^{\mathrm{T}}(t)\right\|-1\right)
$$

where $\rho^{\mathrm{T}}(t)$ represents the partial transpose of $\rho(t)$, and $\|\cdot\|$ denotes the trace norm. The negativity $\mathcal{N}(\rho)$ is a nonnegative function which quantifies the degree of entanglement in a mixed quantum state $\rho$. It vanishes for separable, classically correlated states, and takes on a maximum value if $\rho$ is a pure, maximally entangled state.

In the trapped atom system the Lamb-Dicke parameter $\eta=k / \sqrt{2 m \omega}(\hbar=1)$ controls the probability of changing the vibrational quantum number $n$ in electronic transitions [8]. For small $\eta$ the photon-recoil energy $k^{2} / 2 m$ is an inconsiderable fraction of the trap vibrational frequency $\omega$. In the Lamb-Dicke regime, $\eta \sqrt{n+1} \ll 1$, only the first sideband transitions, $\Delta n= \pm 1$, are significant, their probability being proportional to $(n+1) \eta^{2}$, where $n$ is the vibrational quantum number. Even outside the Lamb-Dicke regime a rapid removal of vibrational energy of a trapped atom occurs under suitable experimental parameters, i.e., a difference of ac-Stark shifts of the two ground states of the order of $\omega[3,6,9$. Under these conditions, with blue detuning of two counter-propagating lasers, a vibrationally excited atom can be driven into states with mean values $\langle n\rangle$ near zero.

An intricate behavior of the negativity is observed during such a vibrational cooling process, as is shown in Fig. 1. We see a rapid build-up of entanglement, a subsequent exponentially damped oscillation, followed by a precipitous drop and a power law behavior and, finally, a rebirth of entanglement towards the non-equilibrium stationary state.

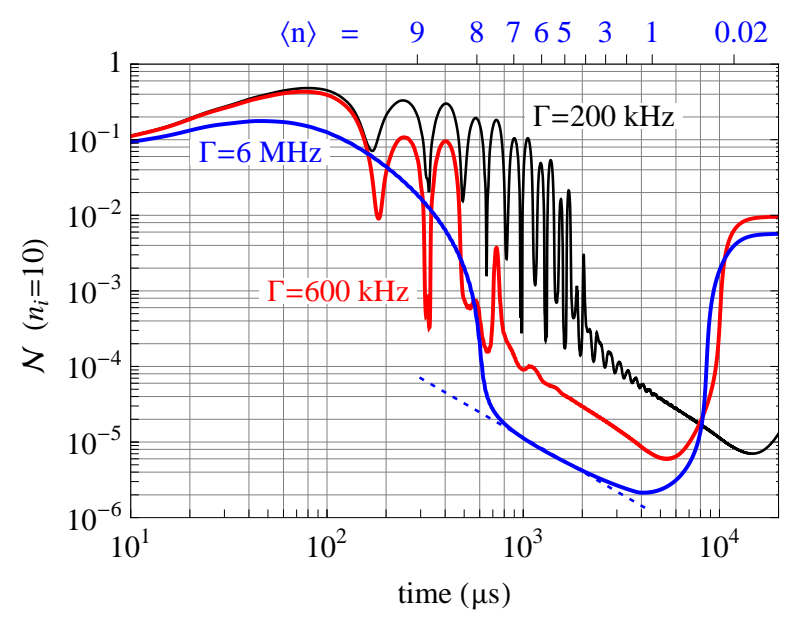

FIG. 1: Dynamics of the negativity during the cooling process for the initial vibrational level $n_{i}=10$ for three values of the excited state width $\Gamma$ and $\eta=0.1$. The top scale gives the mean vibrational quantum number for the $6 \mathrm{MHz}$ result. The dotted line represents a power law decay, $\mathcal{N} \propto t^{-3 / 2}$.

We demonstrate that this complex behavior of the entanglement dynamics can be related to Landau-Zener splittings or avoided crossings in the spectrum of the atom-laser field Hamiltonian. The dominant source of entanglement is thus a near degeneracy of vibronic states, which appears as a consequence of the ac-Stark shift, suitably chosen for EIT-cooling. Two-photon transitions associated with a change in vibrational quantum number occur and lead to a periodic oscillation of entanglement. In addition, we derive an effective Hamiltonian that al- 
lows the modeling of the negativity decay in the presence of dissipation induced by spontaneous emission processes.

Our model is based on the quantum master equation

$$
\frac{d}{d t} \rho(t)=-\mathrm{i}\left[H^{\eta}, \rho(t)\right]+\mathcal{L} \rho(t)
$$

for the interaction picture density matrix $\rho(t)$ representing the combined state of vibrational and electronic degrees of freedom of the trapped atom. The total Hamiltonian

$$
H^{\eta}=H_{\mathrm{cm}}+H_{\mathrm{el}}+H_{\mathrm{int}}^{\eta}
$$

of the model consists of three parts: $H_{\mathrm{cm}}=\omega a^{\dagger} a$ describes the vibrational degree of freedom of a harmonically trapped atom with raising and lowering operators $a^{\dagger}$ and $a$, and $H_{\mathrm{el}}=\Delta(|1\rangle\langle 1|+| 2\rangle\langle 2|)$ represents the electronic degree of freedom with detuning $\Delta$. The electronic states are denoted by $|i\rangle, i=1,2,3$, and form a $\Lambda$-type level structure sketched in Fig. 2a. The Hamiltonian
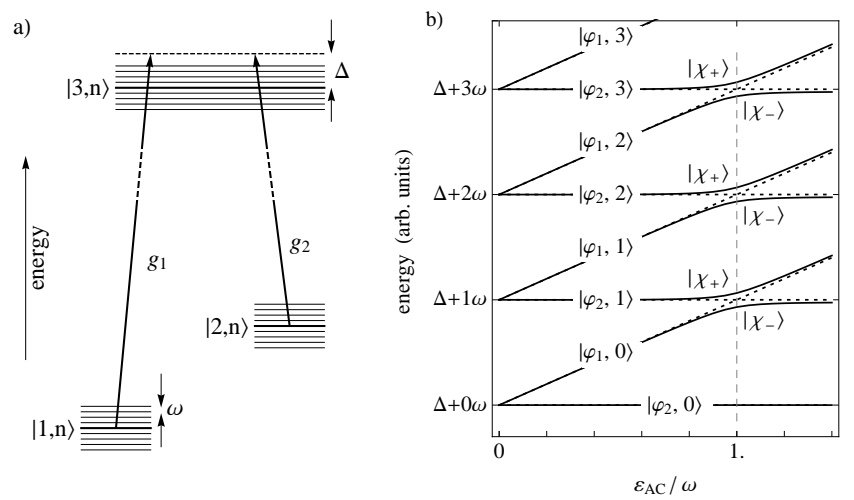

FIG. 2: a) Trapped 3-level atom, b) energies of dressed vibronic states as a function of the normalized ac-Stark shift.

$$
H_{\mathrm{int}}^{\eta}=\frac{g_{1}}{2} e^{i k x}|3\rangle\left\langle 1\left|+\frac{g_{2}}{2} e^{-i k x}\right| 3\right\rangle\langle 2|+\text { h.c. }
$$

models the interaction with two counter-propagating laser beams, the most efficient configuration for cooling, where $x$ denotes the atomic position and $k$ the wavenumber $\left(k_{1} \approx k_{2}=k\right)$. Note that $H_{\text {int }}^{\eta}$ depends on the LambDicke parameter $\eta$ through $k x=\eta\left(a^{\dagger}+a\right)$. Finally, dissipation caused by spontaneous emissions is modelled by the Lindblad superoperator (see, e.g., Ref. [10])

$$
\mathcal{L} \rho=\sum_{j=1,2} \sum_{q= \pm} \frac{\Gamma}{4}\left[\sigma_{j q} \rho \sigma_{j q}^{\dagger}-\frac{1}{2}\left\{\sigma_{j q}^{\dagger} \sigma_{j q}, \rho\right\}\right]
$$

where $\Gamma$ denotes the spontaneous emission rate and $\sigma_{j+}=|j\rangle\left\langle 3\left|e^{i k x}, \sigma_{j-}=\right| j\right\rangle\langle 3| e^{-i k x}$. The Lindblad operators $\sigma_{j+}$ describe the transitions from the excited electronic state $|3\rangle$ to the ground states $|j=1,2\rangle$ with a photon emitted parallel to the harmonic oscillator axis, while the $\sigma_{j-}$ describe the transitions from $|3\rangle$ to $|j=1,2\rangle$ with a photon emitted counter-propagating to the harmonic oscillator axis. This is a good compromise short of doing a full 3D simulation as the recoil of the translational motion is zero for emissions perpendicular to the trap axis, while it leads to Doppler heating or cooling for emissions along the trap axis. Typical results obtained by numerically solving Eq. (2) 3, 6, are given in Fig. 1.

We start our discussion of the entanglement dynamics by considering first the case $\Gamma=0$, neglecting completely effects from spontaneous emission processes. The dynamics is then given by the Schrödinger equation

$$
\frac{d}{d t}|\psi(t)\rangle=-\mathrm{i} H^{\eta}|\psi(t)\rangle .
$$

Our goal is to describe the basic features of the dynamical behavior of the negativity in terms of the properties of the spectrum of the full Hamiltonian $H^{\eta}$ given by Eq. (3). For vanishing Lamb-Dicke parameter, $\eta=0$, the external and the internal degrees of freedom of the atom decouple, such that the eigenstates of $H^{0}=H_{\mathrm{cm}}+H_{\mathrm{el}}+H_{\mathrm{int}}^{0}$ are given by tensor product states $\left|\varphi_{i}, n\right\rangle \equiv\left|\varphi_{i}\right\rangle \otimes|n\rangle$ with corresponding energy eigenvalues, $\varepsilon_{i, n}=\varepsilon_{i}+n \omega$. Here, $n=0,1,2, \ldots$ denotes the vibrational quantum number. The $\left|\varphi_{i}\right\rangle$ are dressed electronic states which are defined as normalized eigenstates of $H_{\mathrm{el}}+H_{\mathrm{int}}^{0}$ with the eigenvalues

$$
\varepsilon_{1}=\Delta+\varepsilon_{\mathrm{AC}}, \quad \varepsilon_{2}=\Delta, \quad \varepsilon_{3}=-\varepsilon_{\mathrm{AC}} .
$$

where $\varepsilon_{\mathrm{AC}}=\frac{1}{2}\left(\sqrt{\Delta^{2}+g_{1}^{2}+g_{2}^{2}}-\Delta\right)$ is the ac-Stark shift. Note that we have labelled the dressed states such that for $g_{1} \gg g_{2}$ the state $\left|\varphi_{i}\right\rangle$ has the main weight on the electronic state $|i\rangle$, and that $\left|\varphi_{2}\right\rangle$ represents the dark state which decouples from the laser fields for $\eta=0$. It follows from the above equations that the levels $\varepsilon_{2, n}$ and $\varepsilon_{1, n-1}$ cross each other (see Fig. $2 \mathrm{~b}$ ) if the resonance condition

$$
\omega=\varepsilon_{\mathrm{AC}} \approx \frac{g_{1}^{2}+g_{2}^{2}}{4 \Delta}
$$

is satisfied. The approximation in Eq. (8) refers to the case $g_{1,2} \ll \Delta$ which is assumed throughout the paper.

For non-vanishing Lamb-Dicke parameter, $\eta>0$, the electronic and the translational degrees of freedom of the atom are coupled. This coupling lifts the degeneracy of the levels $\varepsilon_{2, n}$ and $\varepsilon_{1, n-1}$ at the crossing point to yield an avoided crossing or Landau-Zener splitting [11-13] with a certain energy gap $\Delta E_{n}$, as is illustrated in Fig. 2 b.

Employing degenerate perturbation theory it can be shown that the eigenstates at the center of the avoided crossing are given by the even and odd linear combinations of the unperturbed (crossing) states. More precisely, this means that with an appropriate choice of the phase of the states $\left|\varphi_{2}, n\right\rangle$ and $\left|\varphi_{1}, n-1\right\rangle$ the eigenstates of the full Hamiltonian at resonance have the approxi- 
mate form

$$
\left|\chi_{ \pm}\right\rangle=\frac{1}{\sqrt{2}}\left(\left|\varphi_{2}\right\rangle \otimes|n\rangle \pm\left|\varphi_{1}\right\rangle \otimes|n-1\rangle\right)
$$

with the corresponding energy eigenvalues

$$
E_{ \pm}=\Delta+n \omega \pm \frac{\Delta E_{n}}{2} .
$$

Considering as initial state $|\psi(0)\rangle=\left|\varphi_{2}, n\right\rangle$, it follows that the solution of the Schrödinger equation is given by

$$
\begin{aligned}
|\psi(t)\rangle & =\frac{1}{\sqrt{2}}\left(e^{-i E_{+} t}\left|\chi_{+}\right\rangle+e^{-i E_{-} t}\left|\chi_{-}\right\rangle\right) \\
& =\frac{1}{2}\left(e^{-i E_{+} t}+e^{-i E_{-} t}\right)\left|\varphi_{2}\right\rangle \otimes|n\rangle \\
& +\frac{1}{2}\left(e^{-i E_{+} t}-e^{-i E_{-} t}\right)\left|\varphi_{1}\right\rangle \otimes|n-1\rangle .
\end{aligned}
$$

The Schmidt coefficients [14] of this state take the form

$$
\alpha_{1,2}=\frac{1}{2}\left|e^{-i E_{+} t} \pm e^{-i E_{-} t}\right|,
$$

which immediately leads to the negativity:

$$
\mathcal{N}(t)=\alpha_{1} \alpha_{2}=\frac{1}{2}\left|\sin \left(\Delta E_{n} \cdot t\right)\right|
$$

The same formula is obtained if one considers the initial state $|\psi(0)\rangle=\left|\varphi_{1}, n-1\right\rangle$. Thus, we see that the entanglement dynamics is determined by a single spectral quantity, namely the energy gap $\Delta E_{n}$, describing an oscillation of the negativity with the period $T=\pi / \Delta E_{n}$. In Fig. 3 we compare the negativity obtained from the numerical solution of the full Schrödinger equation (6)
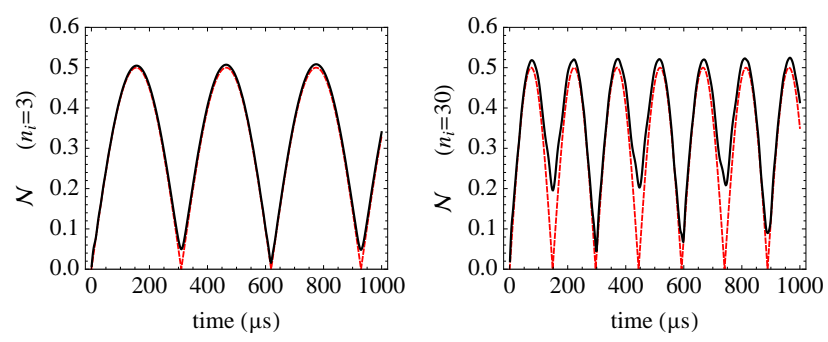

FIG. 3: Time dependence of the negativity obtained from the Schrödinger equation (6) for initial vibrational levels $n_{\mathrm{i}}=3$ and 30. Parameters: $\eta=0.1, \omega=0.03, \Delta=15, g_{1}=1.34$, $g_{1} / g_{2}=10$ (all frequencies are given in units of $2 \pi \mathrm{MHz}$ ). The broken lines represent the prediction according to Eq. 13.

with the formula 13 for various initial states, which clearly demonstrates the accuracy of the approximation based on a single Landau-Zener state pair (9). The negativity predicted by Eq. (6) slightly exceeds the value $1 / 2$ as it also accounts for the small contributions to entanglement by the excited state levels $\left|\varphi_{3}, n\right\rangle$. We emphasize that the argument which leads to the result 13 is quite general and should be applicable to many different physical scenarios: It only requires the existence of a Landau-Zener splitting between tensor product states on some composite Hilbert space. Moreover, depending only on the energy gap, formula $(13)$ can be applied even if the structure of the factors of the tensor products is not known.

Next we discuss the influence of dissipation on the entanglement dynamics which is described by the Lindblad dissipator of Eq. (5). Figure 1 shows examples for the negativity dynamics obtained from numerical solutions of the full master equation (2). We see that the negativity follows a damped oscillation for small $\Gamma$, where the revivals of the negativity come in pairs of nearly equal heights. At a certain threshold value of $\Gamma$ the oscillations die out and a monotonic decay of the negativity after the first bump is observed.

The entanglement behavior under dissipation can be modelled through the Schrödinger equation

$$
\frac{d}{d t}|\psi(t)\rangle=-\mathrm{i} H_{\mathrm{eff}}^{\eta}|\psi(t)\rangle,
$$

with the effective Hamiltonian

$$
H_{\text {eff }}^{\eta}=H^{\eta}-\mathrm{i} \frac{\Gamma}{2}|3\rangle\langle 3| .
$$

This is a non-Hermitian Hamiltonian derived from the quantum master equation (2): It describes the coherent motion generated by $H^{\eta}$ as well as the loss term (anticommutator) of the dissipator (5) which leads to finite widths of the dressed states of the atom-field system given by the imaginary parts of the complex eigenvalues. Employing the effective Schrödinger equation (14) we thus neglect the gain terms of the dissipator (5). These latter terms describe real emission processes and induce, at least partially, transitions into other LandauZener state pairs which add incoherently and, thus, lead only to negligible contributions to the negativity.

In the framework of this approximation we can extend the Landau-Zener state pair calculation to the effective Hamiltonian 15. Under the conditions $g_{1,2} \ll \Delta$ and $\Gamma \ll \Delta$ the eigenvalues of $H_{\mathrm{eff}}^{0}$ are

$$
\begin{aligned}
& \varepsilon_{1}=\Delta+\frac{g_{1}^{2}+g_{2}^{2}}{4 \Delta}-\mathrm{i} \frac{\gamma_{1}}{2}, \\
& \varepsilon_{2}=\Delta, \\
& \varepsilon_{3}=-\frac{g_{1}^{2}+g_{2}^{2}}{4 \Delta}-\frac{\mathrm{i}}{2}\left(\Gamma-\gamma_{1}\right),
\end{aligned}
$$

where $\gamma_{1}=\Gamma\left(g_{1}^{2}+g_{2}^{2}\right) /\left(4 \Delta^{2}\right)$. We denote the eigenstates corresponding to the eigenvalues $\varepsilon_{1,2}$ again by $\left|\varphi_{1,2}\right\rangle$, and the Landau-Zener pair by $\left|\varphi_{1}, n-1\right\rangle$ and $\left|\varphi_{2}, n\right\rangle$. Using these states as basis states, the effective Hamiltonian (15) reduces within the two-state resonance approximation to the matrix

$$
H_{\mathrm{eff}}^{\eta}=\left(\begin{array}{cc}
\Delta+n \omega-\mathrm{i} \gamma_{1} / 2 & \Delta E_{n} / 2 \\
\Delta E_{n} / 2 & \Delta+n \omega
\end{array}\right) .
$$


This Hamiltonian may be viewed as describing damped Rabi-type oscillations between the Landau-Zener pair states with Rabi frequency $\Delta E_{n}$, where only the state $\left|\varphi_{1}, n-1\right\rangle$ is damped, since the width of the dark state $\left|\varphi_{2}, n\right\rangle$ is practically zero. With the effective Hamiltonian (19) it is now easy to determine the solution of the Schrödinger equation (14) corresponding to the initial state $|\psi(0)\rangle=\left|\varphi_{2}, n\right\rangle$, and to derive for the negativity,

$$
\mathcal{N}(t)=\frac{\Delta E_{n}}{2}\left|\frac{\sin \nu t}{\nu}+\frac{\gamma_{1}}{2 \nu^{2}}(1-\cos \nu t)\right| e^{-\gamma_{1} t / 2},
$$

where $\nu=\sqrt{\left(\Delta E_{n}\right)^{2}-\gamma_{1}^{2} / 4}$. As is illustrated in Fig. 4 the simple formula 20 provides an excellent approximation of the entanglement dynamics for $\gamma_{1} / 2<\Delta E_{n}$, and even for intermediate values of the spontaneous emission rate the qualitative behavior of the negativity is reproduced. It is remarkable that Eq. 20 correctly describes the transition from the underdamped to the overdamped motion of the negativity at $\gamma_{1} / 2=\Delta E_{n}$, compare Figs. 4k and $4 \mathrm{~d}$. Using the approximations $\Delta E_{n} \approx \eta \sqrt{n} g_{1} g_{2} / \Delta$ and $\gamma_{1} \approx \Gamma g_{1}^{2} /\left(4 \Delta^{2}\right)$ this transition is predicted to occur at the value $\Gamma=8 \eta \sqrt{n} \Delta g_{2} / g_{1}$, which nicely fits to the results of our numerics.
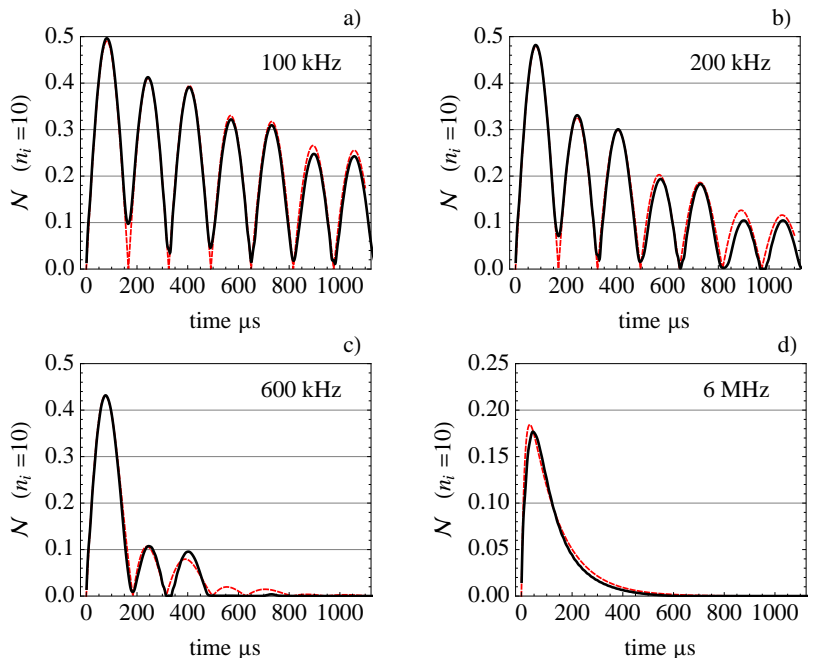

FIG. 4: The negativity obtained from the quantum master equation 2 for various $\Gamma$, as also shown in Fig. 1 Fits of Eq. 20 are shown by the broken lines. Conditions as given in Fig. 3 .

The long-time behavior of the negativity for larger values of the spontaneous emission rate $\Gamma$ exhibits an interesting and complex structure, see Fig. 1. The exponential decay of the initial peak of the negativity is followed by a precipitous drop until the rate of decay slows down and a smooth transition to an algebraic decay of the form $\mathcal{N}(t) \propto t^{-3 / 2}$ emerges, see the broken line in Fig. 1. While a detailed discussion is beyond the scope of this note, we mention that the Landau-Zener picture can also offer an explanation here. A power law gov- erns the negativity dynamics until a rebirth of entanglement towards the final value $\mathcal{N}(\infty)=\mathcal{N}\left(\rho_{\text {stat }}\right)$ occurs. The final value of the negativity represents the entanglement in the unique non-equilibrium stationary state $\rho_{\text {stat }}$ of the master equation (2). During the cooling the atom is driven into a mixed stationary state which has a dominant contribution from the state $\left|\varphi_{2}, 0\right\rangle$, the product of the dark state and the vibrational ground state of the trap. However, $\rho_{\text {stat }}$ contains a small amount of entanglement which is controlled by the small steadystate heating caused by off-diagonal transitions from the dark state. Also here the LZ-model offers a quantitative explanation. The interaction of the lowest dark state, $\left\langle\varphi_{2}, 0\left|H_{\text {int }}^{\eta}\right| \varphi_{1}, 1\right\rangle=\eta g_{1} g_{2} /(4 \Delta)$, leads to a steady state negativity, $\mathcal{N}(\infty)=\eta g_{2} / g_{1}$ for $\Gamma \rightarrow 0$, in agreement with the predictions from Eq. 2 for the examples in Fig. 1 .

The Landau-Zener mechanism suggest various methods for the control of entanglement between electronic and translational degrees of freedom of trapped atoms by means of suitable laser fields. One possibility is the switching of fields at appropriate points of time, e.g., at the instant of maximal entanglement. Since the manifold spanned by the Landau-Zener state pair has only a negligible contribution from the excited electronic state, the negativity practically freezes upon rapidly switchingoff the lasers. This method can thus create permanent entanglement of the trapped atom. A further possibility is to use suitable laser pulses in order to generate entanglement through the control of adiabatic/diabatic Landau-Zener transitions. A detailed discussion of these points will be given in a forthcoming paper.

This work was supported by the Deutsche Forschungsgemeinschaft (Grant HE-2525/8).

[1] C. F. Roos et al., Phys. Rev. Lett. 85, 5547 (2000).

[2] F. Schmidt-Kaler et al., Appl. Phys. B 73, 807 (2001).

[3] M. Roghani, H. P. Breuer and H. Helm, Phys. Rev. A81, 033418 (2010).

[4] F. Mintert et al., Phys. Rep. 415, 207 (2005).

[5] M. Roghani, H. Helm, and H. P. Breuer, Physica Scripta T140, 014034 (2010).

[6] M. Roghani and H. Helm, Phys. Rev. A77, 43418 (2008).

[7] G. Vidal and R.F. Werner, Phys. Rev. A65, 32314 (2002).

[8] M. Lewenstein et al., Phys. Rev. A50, 2207(1994).

[9] G. Morigi, J. Eschner, and C. Keitel, Phys. Rev. Lett. 85, 4458 (2000).

[10] H. P. Breuer and F. Petruccione, The Theory of Open Quantum Systems (Oxford University Press, Oxford, 2007).

[11] L. D. Landau, Phys. Z. Sowjetunion 2, 46 (1932).

[12] C. Zener, Proc. R. Soc. A137, 696 (1932).

[13] E. C. G. Stückelberg, Helv. Phys. Acta 5, 369 (1932).

[14] I. Bengtsson and K. Życzkowski, Geometry of Quantum States: An Introduction to Quantum Entanglement (Cambridge University Press, Cambridge, 2006). 